\title{
Dynamics of a supercritical composite shaft mounted on viscoelastic supports
}

\author{
O. Montagnier ${ }^{\mathrm{a}, \mathrm{b}, *}$, C. Hochard ${ }^{\mathrm{c}}$ \\ ${ }^{a}$ Centre de recherche de l'Armée de l'air (CReA), Écoles des Officiers de l'Armée de l'Air (EOAA), BA 701, F-13361 Salon Air, France \\ ${ }^{b}$ LMA, CNRS, UPR 7051, F-13402 Marseille Cedex 20, France \\ ${ }^{c}$ LMA, CNRS, UPR 7051, Aix-Marseille Univ, Centrale Marseille, F-13402 Marseille Cedex 20, France
}

\begin{abstract}
The damping in a carbon fiber reinforced plastic (CFRP) laminate is greater than that which occurs in most metallic materials. In the supercritical regime, the damping can trigger unstable whirl oscillations, which can have catastrophic effects. The vibrations occurring in a supercritical composite drive shaft are investigated here in order to predict instabilities of this kind. A simply supported carbon/epoxy composite tube mounted on viscoelastic supports is studied, using an approximation of the Rayleigh-Timoshenko equation. The damping process is assumed to be hysteretic. The composite behavior is described in terms of modulus and loss factor, taking homogenized values. The critical speeds are obtained in several analytical forms in order to determine the effects of factors such as the rotatory inertia, the gyroscopic forces, the transverse shear and the supports stiffness. Assuming that the hysteretic damping can be expressed in terms of the equivalent viscous model, the threshold speed is obtained in the form of an analytical criterion. The influence of the various factors involved is quantified at the first critical speed of a subcritical composite shaft previously described in the literature. The influence of the coupling mechanisms on the unsymmetrical composite laminate and the end fittings is also investigated using a finite element model. None of these parameters were found to have a decisive influence in this case. Those having the greatest effects were the transverse shear and the supports stiffness. The effects of the composite stacking sequence, the shaft length and the supports stiffness on the threshold speed were then investigated. In particular, drive shafts consisting only of $\pm 45^{\circ}$ or $\pm 30^{\circ}$ plies can be said to be generally unstable in the supercritical regime due to their very high loss factors.
\end{abstract}

Keywords: composite shaft, drive shaft, rotating damping, hysteretic damping, critical speed, threshold speed

\section{Introduction}

The use of driveshafts in the supercritical regime has proved to be of great interest in many applications, especially those involving long drivelines (helicopters, tilt rotors, etc.). However, in the field of rotor dynamics, internal damping, which is also called rotating damping, is known to cause whirl instabilities in this speed regime. In particular, with long driveshafts consisting of materials which are more dissipative than metallic materials (such as most CFRP laminates [1]), these instabilities tend to occur more frequently. The aim of the present study was to develop a theoretical model for predicting these instabilities and to establish the most decisive physical parameters involved.

${ }^{*}$ Corresponding author. Tel. : $(+33) 4.90 .17 .80 .93$; fax : $(+33) 4.90 .17 .81 .89$.

Email address: olivier.montagnier@inet.air.defense.gouv.fr (O. Montagnier) 


\begin{tabular}{|c|c|c|c|}
\hline \multicolumn{2}{|c|}{ Nomenclature } & \multirow{2}{*}{$\begin{array}{l}\varepsilon^{0} \\
\varepsilon\end{array}$} & \multirow{2}{*}{$\begin{array}{l}\text { strain field vector of the laminate middle plane } \\
\text { shaft eccentricity }\end{array}$} \\
\hline $\mathbf{A}, \mathbf{B}, \mathbf{D}$ & in-plane, coupling and bending stiffness matrix & & \\
\hline & of the laminate & $\eta$ & loss factor matrix \\
\hline$c$ & viscous damping & $\eta$ & loss factor \\
\hline$d$ & modal damping or decay rate & $\theta$ & out-of plane cross-section rotation (complex or \\
\hline E & longitudinal Young's modulus of the shaft & & real) \\
\hline$E_{11}, E_{22}$ & longitudinal and transverse Young's modulus, & $\Theta$ & complex amplitude of the complex rotation $\theta$ \\
\hline \multirow[t]{2}{*}{$v_{12}, G_{12}$} & Poisson's ratio and in-plane shear modulus of & $\kappa$ & shear coefficient \\
\hline & the ply & $\lambda$ & shaft complex frequency \\
\hline$E^{*}, G^{*}$ & complex modulus & $v$ & Poisson's ratio \\
\hline$f$ & oscillation frequency & $\rho$ & shaft mass density \\
\hline$G$ & transverse shear modulus of the shaft & $\sigma$ & stress field \\
\hline$h$ & height of the layer & $\omega$ & natural frequency of the flexural modes \\
\hline$I_{x}, I_{y}, I_{z}$ & $\begin{array}{l}\text { polar and transverse area moment of inertia of } \\
\text { the shaft }\end{array}$ & $\Omega$ & spin speed \\
\hline $\mathrm{i}$ & imaginary unit $\sqrt{-1}$ & Subscript & \\
\hline $\mathbf{k}$ & curvature field vector & $B-, B+$ & lower and higher backward whirl speeds \\
\hline$k$ & stiffness & $b$ & bearing \\
\hline$l$ & shaft length (between the two supports) & $c$ & critical \\
\hline $\mathbf{M}$ & bending moment vector in the laminate & $e$ & external \\
\hline$m$ & mass & eq & equivalent \\
\hline $\mathbf{N}$ & membrane force vector in the laminate & $F-, F+$ & lower and higher forward whirl speeds \\
\hline$n$ & number of sine modes or number of harmonic & $i$ & internal \\
\hline$p$ & number of composite layers & $m$ & medium \\
\hline $\mathbf{Q}$ & in-plane stiffness matrix of a ply & $p$ & $\in\{B-, B+, F-, F+\}$ \\
\hline$r$ & shaft radius & $s$ & shaft \\
\hline$S$ & shaft cross-section area & th & threshold speed \\
\hline$t$ & thickness & 0 & gyroscopic effects assumed to be negligible \\
\hline$u$ & displacement (complex or real) & & $\left(\Gamma_{n} \approx 0\right)$ \\
\hline \multirow[t]{2}{*}{$U$} & complex amplitude of the complex & & \\
\hline & displacement $u$ & Superscript & \\
\hline$\widehat{U_{\mathrm{el}}}$ & Elastic strain energy per surface unit & $T$ & transpose \\
\hline $\mathbf{x}, \mathbf{y}, \mathbf{z}$ & coordinates & - & order of magnitude of a quantity \\
\hline$\varepsilon$ & strain field vector & & \\
\hline
\end{tabular}

The instabilities mentioned above can be counterbalanced by applying sufficiently strong external damping and/or using the effect of suspension anisotropy $[2,3]$. There exist several passive ways for increasing this non-rotating damping. Most turbines are constructed with hydrodynamic bearings or squeeze-film dampers, but the main disadvantages of these devices are their cost, their complexity and the additional instabilities they induce. Rolling-element bearings do not destabilize rotors but provide insufficient damping. During the last twenty years, dissipative materials such as elastomers have emerged as suitable materials for bearing suspensions [4-6]. Here it is proposed to study this low cost configuration consisting of an axisymmetric composite shaft simply supported on classical rolling-element bearings mounted on viscoelastic materials (Fig. 1).

Various approaches based on beam and shell theories have been presented for determining the critical speeds of an ax-

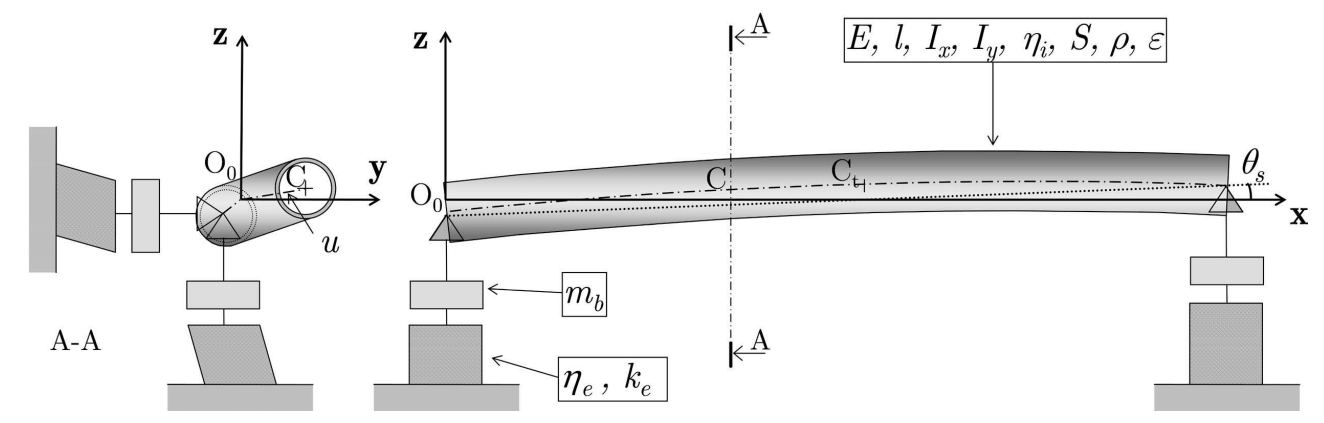

Figure 1: Simply supported axisymmetric tubular composite shaft with rolling-element bearings mounted on viscoelastic supports. 
Table 1: Material properties for a 0.6 volume fraction

\begin{tabular}{lllllll}
\hline Material & Abbr. & $\rho\left(\mathrm{kg} \mathrm{m}^{-3}\right)$ & $E_{11}(\mathrm{GPa})$ & $E_{22}(\mathrm{GPa})$ & $G_{12}(\mathrm{GPa})$ & $v_{12}$ \\
\hline Gubran et al. [12] & $\mathrm{CE}_{\mathrm{G}}{ }^{a}$ & 1500 & 130 & 10 & 7.0 & 0.25 \\
Narmco 5505 [14] & $\mathrm{BE}^{b}$ & 1965 & 211 & 24.1 & 6.9 & 0.36 \\
T300/5208 [15, 16] & $\mathrm{CE}_{\mathrm{L} .}$ & 1680 & 181 & 10.3 & 7.17 & 0.1321 \\
\hline
\end{tabular}

isymmetric tubular composite shaft mounted on elastic or infinitely rigid supports [7-13]. The simplest of these approaches is called the Equivalent Modulus Beam Theory (EMBT) [14]. This method involves calculating the eigenvalues of the isotropic Bernoulli beam, using the longitudinal modulus of the composite material computed with the classical laminate theory (LT). Several authors have established the accuracy of this method in the case of symmetric laminates, but the EMBT approach has proved to have some limitations in the case of unbalanced and unsymmetrical laminates, as summarized below. The EMBT does not take into account the ply location relative to the axis when dealing with multilayered unsymmetrical laminates. However, Gubran and Gupta [12] have established that the difference in the natural frequencies amount to only $5 \%$ in the most extreme cases, i.e., $\left[0^{\circ}, 90^{\circ}\right]$ versus $\left[90^{\circ}, 0^{\circ}\right]$ (shaft properties: $l=1 \mathrm{~m}, t_{s}=4 \mathrm{~mm}$ and $r_{m}=50 \mathrm{~mm}$ and $C E_{\mathrm{G}}$, see Table 1 and the list of nomenclature for the symbol definitions). This difference is greater in the case of shafts with small diameters. The EMBT does not take shear-normal coupling into account in the case of unbalanced laminates, or bending-stretching and bending-twisting coupling in that of unsymmetrical laminates $[10,12]$. However, the numerical analysis presented in this paper shows that the latter two effects are negligible in the case of composite tubes, due to the tubular structure (see 3.1.5). Furthermore, the beam theories cannot take into account of the effect done by the centrifugal forces associated with the deformation. Martínez-Casas et al. [17] have established with a shell theory that this effect can increase the first natural frequency by about $0.5 \%$ on a steel cylinder (shaft properties: $l=1.5 \mathrm{~m}, r=50 \mathrm{~mm}, \rho=7800 \mathrm{~kg} \mathrm{~m}^{-3}$, $E=210 \mathrm{GPa}$ and $v=0.3$ ). An EMBT was developed here, in which transverse shear, rotatory inertia and gyroscopic effect were taken into account. The significance of these factors is discussed in the last part of the paper (see 3.1).

The internal damping resulting from dissipation in the shaft material and dry friction between assembled components is conventionally included in the governing equations using the viscous damping model. However, most materials such as CFRP composites undergo a damping which resembles hysteretic damping much more than viscous damping $[1,18,19]$. The main characteristic of hysteretic damping is that the corresponding stress-strain loop is independent of the excitation frequency. It is generally defined in terms of the complex modulus $E^{*}=E(1+\eta)$, where $\eta$ is the loss factor. However, since it is not easy to introduce the complex model into rotor dynamic equations, it is necessary to use the classical equivalence with the viscous damping model [20-22]. In the present analysis, all the internal damping is assumed to be hysteretic. This approach has been called the equivalent complex modulus beam theory (ECMBT), by analogy with EMBT. The model developed here was previously studied for use with isotropic materials and with Bernoulli assumptions in [6].

The first part of this paper deals with the composite shaft dynamics. The critical speeds are calculated in various forms, depending on the assumptions. The threshold speed is then obtained with an analytical criterion. The procedure used to determine the equivalent modulus and the equivalent damping of the shaft is described. In the second part, the case of the composite shaft studied by Zinberg and Symonds [14] is investigated. The results obtained are compared with data previously published in the literature. The aim of this numerical analysis is to assess the contribution of the main factors such as the rotatory inertia, the gyroscopic forces, the transverse shear and the supports stiffness to the critical speeds. The effects of the coupling mechanisms in the unsymmetrical composite laminate and those of the end fittings are then studied by performing finite element (FE) simulations on ABAQUS [23]. In the last part, the critical speeds and the threshold speed of composite shafts with internal hysteretic damping are computed with several stacking sequences. Lastly, the effects of the shaft length and the supports stiffness on the stability are investigated.

\section{Rotordynamic analysis}

\subsection{Governing equations}

The dynamic properties of the simply supported composite drive shaft mounted on viscoelastic supports shown in Fig. 1 were studied. The bearings, which are assumed to be infinitely rigid in comparison with the supports, are simply modeled in terms of their mass $m_{b}$. The supports are assumed to be axisymmetric and are accounted for in terms of their stiffness $k_{e}$ and their loss factor $\eta_{e}$. For the sake of convenience, equivalent external and internal viscous damping terms $c_{e}$ and $c_{i}$ will be used in the equations.

The displacements will be expressed in the complex form. The cross-sectional displacement, i.e., the displacement of the shaft section center (denoted C in Fig. 1) relative to the fixed frame, is composed of a rigid-body displacement $u_{b}=u_{b y}+\mathrm{i} u_{b z}$ 
(cylindrical whirl), a rigid-body rotation $\theta_{b}=\theta_{b y}+\mathrm{i} \theta_{b z}$ (conical whirl) and a deflection of the shaft $u_{s}=u_{s y}+\mathrm{i} u_{s z}$. It can be written as follows:

$$
u(x, t)=u_{b}(t)+\left(x-\frac{l}{2}\right) \theta_{b}(t)+u_{s}(x, t)
$$

Note that $u^{\prime \prime}=u_{s}^{\prime \prime}$ where ()$^{\prime}=\partial / \partial x$. The cross-sectional rotation relative to the fixed frame is composed of the rigidbody rotation $\theta_{b}$ and the bending rotation $\theta_{s}=\theta_{s y}+\mathrm{i} \theta_{s z}$. It can be written as follows:

$$
\theta(x, t)=\theta_{b}(t)+\theta_{s}(x, t)
$$

With the above assumptions, using Timoshenko equations for the flexural vibration of beams [24] and adding the gyroscopic components, the equations of motion for an axisymmetrical shaft (where $I_{y}=I_{z}$ in particular) are:

$$
\begin{gathered}
\rho S \ddot{u}+\kappa S G\left(\theta_{s}^{\prime}-u_{s}^{\prime \prime}\right)=0 \\
\rho I_{y} \ddot{\theta}-\mathrm{i} \Omega I_{x} \dot{\theta}-E I_{y} \theta_{s}^{\prime \prime}+\kappa S G\left(\theta_{s}-u_{s}^{\prime}\right)=0
\end{gathered}
$$

where $\left(^{*}\right)=\partial / \partial t, \rho=m_{s} / S l$ is the mass density and $\Omega$ is the spin speed. Note that the terms involving the shaft inertia depend on $u$ and $\theta$, while those involving the rigidity depend on $u_{s}$ and $\theta_{s}$. We can now differentiate Eq. (4),

$$
\rho I_{y} \ddot{\theta}^{\prime}-\mathrm{i} \Omega \rho I_{x} \dot{\theta}^{\prime}-E I_{y} \theta_{s}^{\prime \prime \prime}+\kappa S G\left(\theta_{s}^{\prime}-u_{s}^{\prime \prime}\right)=0
$$

and we write Eq. (3) in the following form:

$$
\theta^{\prime}=\theta_{s}^{\prime}=u_{s}^{\prime \prime}-\frac{\rho}{\kappa G} \ddot{u}
$$

Replacing $\theta^{\prime}$ and $\theta_{s}^{\prime}$ in Eq. (5) by the above equation, we obtain the Rayleigh-Timoshenko equation for a rotating shaft:

$$
\ddot{u}-\frac{I_{y}}{S}\left(1+\frac{E}{\kappa G}\right) \ddot{u}_{s}^{\prime \prime}+\mathrm{i} \Omega \frac{I_{x}}{S} \dot{u}_{s}^{\prime \prime}+\frac{\rho I_{y}}{\kappa S G} \dddot{u}-\frac{\mathrm{i} \Omega \rho I_{x}}{\kappa S G} \dddot{u}+\frac{E I_{y}}{\rho S} u_{s}^{\prime \prime \prime \prime}=0
$$

In the case of long shafts $(l / r \geq 10)$, a dimensional analysis shows that the terms corresponding to $\dddot{u}$ and $\dddot{u}$ in the above equation are small. Let us take the notation ${ }^{-}$to denote the order of magnitude of a quantity. The orders of magnitude of the area moment of inertia $(I)$ and the surface $(S)$ are $\bar{t} \bar{r}^{3}$ and $\bar{t} \bar{r}$, respectively. Let us divide the Rayleigh-Timoshenko equation (Eq. (7)) by $\bar{u} \bar{f}^{2}$ to obtain a non-dimensionalized equation. The order of each term in the above equation can be approximated as follows:

$$
\begin{gathered}
\frac{1}{\bar{u} \bar{f}^{2}} \frac{\partial^{2} u}{\partial t^{2}} \sim 1 \\
\frac{1}{\bar{u} \bar{f}^{2}} \frac{I_{y}}{S}\left(1+\frac{E}{\kappa G}\right) \frac{\partial^{4} u_{s}}{\partial t^{2} \partial^{2} x} \sim \frac{\bar{E}}{\bar{\kappa} \bar{G}}\left(\frac{\bar{r}}{\bar{l}}\right)^{2} \\
\frac{1}{\bar{u} \bar{f}^{2}} \Omega \frac{I_{x}}{S} \frac{\partial^{3} u_{s}}{\partial t \partial^{2} x} \sim\left(\frac{\bar{r}}{\bar{l}}\right)^{2} \\
\frac{1}{\bar{u} \bar{f}^{2}} \frac{\rho I_{y}}{\kappa S G} \frac{\partial^{4} u_{s}}{\partial t^{4}} \sim \frac{\bar{\rho} \bar{r}^{2}}{\bar{\kappa} \bar{G}} \bar{f}^{2} \\
\frac{1}{\bar{u} \bar{f}^{2}} \frac{\Omega \rho I_{x}}{\kappa S G} \frac{\partial^{3} u_{s}}{\partial t^{3}} \sim \frac{\bar{\rho} \bar{r}^{2}}{\bar{\kappa} \bar{G}} \bar{f}^{2} \\
\frac{1}{\bar{u} \bar{f}^{2}} \frac{E I_{y}}{\rho S} \frac{\partial^{4} u_{s}}{\partial x^{4}} \sim \frac{\bar{E} \bar{r}^{2}}{\bar{\rho} \bar{l}^{4}} \frac{1}{\bar{f}^{2}}
\end{gathered}
$$

If the shaft is sufficiently long, the oscillation frequency will obviously depend mainly on the first and last terms in the Rayleigh-Timoshenko equation. It can be concluded that these two terms are of a similar order of magnitude:

$$
\frac{1}{\bar{u} \bar{f}^{2}} \frac{E I_{y}}{\rho S} \frac{\partial^{4} u_{s}}{\partial x^{4}} \sim \frac{1}{\bar{u} \bar{f}^{2}} \frac{\partial^{2} u}{\partial t^{2}} \sim 1 \Rightarrow \bar{f} \sim \sqrt{\frac{\bar{E} \bar{r}^{2}}{\bar{\rho} \bar{l}^{4}}}
$$


Let us replace $\bar{f}$ in Eqs. (11) and (12) so as to obtain the order of magnitude of the terms in $\dddot{u}$ and $\dddot{u}$ :

$$
\begin{gathered}
\frac{1}{\bar{u} \bar{f}^{2}} \frac{\rho I_{y}}{\kappa S G} \frac{\partial^{4} u_{s}}{\partial t^{4}} \sim \frac{\bar{E}}{\bar{\kappa} \bar{G}}\left(\frac{\bar{r}}{\bar{l}}\right)^{4} \\
\frac{1}{\bar{u} \bar{f}^{2}} \frac{\mathrm{i} \Omega \rho I_{x}}{\kappa S G} \frac{\partial^{3} u_{s}}{\partial t^{3}} \sim \frac{\bar{E}}{\bar{\kappa} \bar{G}}\left(\frac{\bar{r}}{\bar{l}}\right)^{4}
\end{gathered}
$$

In the case of a composite shaft, the order of magnitude of $E / \kappa G$ is about 10 (exactly 16.3 in the case of the Zinberg and Symonds' shaft [14]). Assuming $1 / \mathrm{r}=10$, the order of magnitude of the second, third, fourth and fifth terms in the Rayleigh-Timoshenko equation (Eq. (7)) are $10^{-1}, 10^{-2}, 10^{-3}$ and $10^{-3}$, respectively. It can be concluded that the terms in $\dddot{u}$ and $\dddot{u}$ are very small in comparison with the others, and that the term in $\ddot{u}^{\prime}$ (the gyroscopic effect) is small. We can then approximate the Rayleigh-Timoshenko equations in the useful form:

$$
\ddot{u}-\frac{I_{y}}{S}\left(1+\frac{E}{\kappa G}\right) \ddot{u}_{s}^{\prime \prime}+\mathrm{i} \Omega \frac{I_{x}}{S}{\dot{u_{s}}}^{\prime \prime}+\frac{E I_{y}}{\rho S} u_{s}^{\prime \prime \prime \prime}=0
$$

The internal damping (relative to $u_{s}$ and not $u$ ) and the unbalanced forces can be added to obtain the local governing equation of motion:

$$
\ddot{u}-\frac{I_{y}}{S}\left(1+\frac{E}{\kappa G}\right) \ddot{u}_{s}^{\prime \prime}+\mathrm{i} \Omega \frac{I_{x}}{S} \dot{u}_{s}^{\prime \prime}+\frac{E I_{y}}{\rho S} u_{s}^{\prime \prime \prime \prime}+\frac{c_{i}}{\rho S l}\left(\dot{u}_{s}-\mathrm{i} \Omega u_{s}\right)=\varepsilon(x) \Omega^{2} \mathrm{e}^{\mathrm{i} \Omega t}
$$

If $E / \kappa G$ is removed, the above equation becomes the Euler-Bernoulli equation investigated in [6].

The boundary conditions for the shaft and the equations governing the bearings and viscoelastic supports can be written:

$$
u_{s}^{\prime \prime}(0, t)=u_{s}^{\prime \prime}(l, t)=0, u_{s}(0, t)=u_{s}(l, t)=0
$$

and

$$
\begin{aligned}
\int_{0}^{l} \rho S \ddot{u} \mathrm{~d} x+2 m_{b} \ddot{u}_{b}+2 c_{e} \dot{u}_{b}+2 k_{e} u_{b} & =\int_{0}^{l} \rho S \varepsilon(x) \Omega^{2} \mathrm{e}^{\mathrm{i} \Omega t} \mathrm{~d} x \\
\int_{0}^{l} \rho S\left(x-\frac{l}{2}\right) \ddot{u} \mathrm{~d} x+2 m_{b} \frac{l^{2}}{4} \ddot{\theta}_{b}+2 c_{e} \frac{l^{2}}{4} \dot{\theta}_{b}+2 k_{e} \frac{l^{2}}{4} \theta_{b} & =\int_{0}^{l} \rho S\left(x-\frac{l}{2}\right) \varepsilon(x) \Omega^{2} \mathrm{e}^{\mathrm{i} \Omega t} \mathrm{~d} x
\end{aligned}
$$

\subsection{Critical speeds}

Solutions for free motion (Eqs. (18-21) with $\varepsilon=0)$ can be assumed in the following form:

$$
\begin{aligned}
& u_{s}(x, t)=U_{s n} \sin \left(\frac{\pi n x}{l}\right) \mathrm{e}^{\mathrm{i} \lambda_{n} t}, \\
& u_{b}(t)=U_{b n} \mathrm{e}^{\mathrm{i} \lambda_{n} t}, \theta_{b}(t)=\Theta_{b n} \mathrm{e}^{\mathrm{i} \lambda_{n} t}
\end{aligned}
$$

where $n \in \mathbb{N}^{*}$ and $\lambda_{n}$ are the harmonic number and the complex eigenvalues, respectively. Applying the Galerkin principle to Eq. (18) with $\varepsilon=0$ and the trial function (22) (for the detailed procedure, see [6]), we obtain complex characteristic equations of order 4 in $\lambda_{n}$, where the typical $n$th equation is:

$$
\begin{aligned}
\Psi_{n} \lambda_{n}^{4}-\left[\Gamma_{n} \Omega\right. & \left.+\mathrm{i}\left(\Pi_{n} d_{e n}+d_{i n}\right)\right] \lambda_{n}^{3}-\left[\omega_{s n}^{2}+\Pi_{n} \omega_{b n}^{2}+d_{i n} d_{e n}-\mathrm{i} \Omega\left(\Gamma_{n} d_{e n}+d_{i n}\right)\right] \lambda_{n}^{2} \\
& +\left[\left(\Gamma_{n} \omega_{b n}^{2}+d_{i n} d_{e n}\right) \Omega+\mathrm{i}\left(d_{i n} \omega_{b n}^{2}+d_{e n} \omega_{s n}^{2}\right)\right] \lambda_{n}+\left(\omega_{s n}^{2}-\mathrm{i} d_{i n} \Omega\right) \omega_{b n}^{2}=0
\end{aligned}
$$

with

$$
\begin{gathered}
\omega_{s n}=\left(\frac{n \pi}{l}\right)^{2} \sqrt{\frac{E I_{y}}{\rho S}}=\sqrt{\frac{k_{s n}}{m_{s}}}, \omega_{b n}=\sqrt{\frac{k_{e}}{m_{b}+\frac{m_{s}}{2\left(2+(-1)^{n}\right)}}}, d_{e n}=\frac{c_{e}}{m_{b}+\frac{m_{s}}{2\left(2+(-1)^{n}\right)}}=\frac{c_{e} k_{e}}{\omega_{b n}^{2}}, d_{i n}=\frac{c_{i}}{m_{s}}=\frac{c_{i} k_{s n}}{\omega_{s n}^{2}}, \\
\Gamma_{n}=\frac{I_{x}}{S}\left(\frac{n \pi}{l}\right)^{2}, \Pi_{n}=1+\frac{I_{y}}{S}\left(\frac{n \pi}{l}\right)^{2}\left(1+\frac{E}{\kappa G}\right), \Phi_{n}=\frac{m_{s}}{m_{b}+\frac{m_{s}}{2\left(2+(-1)^{n}\right)}}, \Psi_{n}=\Pi_{n}-\frac{4}{n^{2} \pi^{2}} \Phi_{n},
\end{gathered}
$$

where $\omega_{s n}$ is the $n$th natural frequency of the shaft without any rotatory inertia, gyroscopic effect or transverse shear and without any support coupling effects (when the supports are assumed to be infinitely rigid), $\omega_{b 1}$ and $\omega_{b 2}$ are the natural frequencies of the cylindrical rigid-body mode and that of the conical rigid-body mode (when the shaft is assumed to be 
infinitely rigid), respectively. We express the eigenvalue solution in the complex form as follows $\lambda_{n}=\omega_{n}+\mathrm{i} d_{n}$. The real part $\omega_{n}$ and the imaginary part $d_{n}$ are an angular frequency term and a modal damping term, respectively. Hence, assuming the presence of weak damping yields $\omega \gg d$ and in particular, $\left\{\omega_{n}, \omega_{s n}, \omega_{b n}, \Omega\right\} \gg\left\{d_{n}, d_{e n}, d_{i n}\right\}$. This assumption makes it possible to calculate the real part of Eq. (24) at zero order relative to $d$ :

$$
\Psi_{n} \omega_{n}^{4}-\Gamma_{n} \Omega \omega_{n}^{3}-\left(\omega_{s n}^{2}+\Pi_{n} \omega_{b n}^{2}\right) \omega_{n}^{2}+\Gamma_{n} \Omega \omega_{b n}^{2} \omega_{n}+\omega_{b n}^{2} \omega_{s n}^{2} \approx 0
$$

When $\Omega=\omega_{c n}$ or $\Omega=-\omega_{c n}$, Eq. (26) yields the two critical speeds of the forward modes and the two critical speeds of the backward modes in the case of the $n$th harmonic:

$$
\begin{aligned}
& \omega_{c n F \pm}=\frac{1}{\sqrt{2 \Delta_{n-}}} \sqrt{\omega_{s n}^{2}+\Lambda_{n-} \omega_{b n}^{2} \pm \sqrt{\omega_{s n}^{4}+2\left(\Lambda_{n-}-2 \Delta_{n-}\right) \omega_{s n}^{2} \omega_{b n}^{2}+\Lambda_{n-}^{2} \omega_{b n}^{4}}} \\
& \omega_{c n B \pm}=-\frac{1}{\sqrt{2 \Delta_{n+}}} \sqrt{\omega_{s n}^{2}+\Lambda_{n+} \omega_{b n}^{2} \pm \sqrt{\omega_{s n}^{4}+2\left(\Lambda_{n+}-2 \Delta_{n+}\right) \omega_{s n}^{2} \omega_{b n}^{2}+\Lambda_{n+}^{2} \omega_{b n}^{4}}}
\end{aligned}
$$

with

$$
\Delta_{n \pm}=\Psi_{n} \pm \Gamma_{n}, \quad \Lambda_{n \pm}=\Pi_{n} \pm \Gamma_{n}
$$

where \pm stands for the two equations with a positive and negative sign, written in a contracted form. When the supports are assumed to be infinitely rigid, the above equations give the critical speed in the simple form:

$$
\begin{aligned}
\lim _{\omega_{b n} \rightarrow+\infty} \omega_{c n F+} & =+\infty \\
\lim _{\omega_{b n} \rightarrow+\infty} \omega_{c n F-} & =\frac{\omega_{s n}}{\sqrt{1+\frac{I_{y}}{S}\left(\frac{n \pi}{l}\right)^{2}\left(\frac{E}{k G}-1\right)}} \\
\lim _{\omega_{b n} \rightarrow+\infty} \omega_{c n B+} & =-\infty \\
\lim _{\omega_{b n} \rightarrow+\infty} \omega_{c n B-} & =-\frac{\omega_{s n}}{\sqrt{1+\frac{I_{y}}{S}\left(\frac{n \pi}{l}\right)^{2}\left(\frac{E}{k G}+3\right)}}
\end{aligned}
$$

\subsection{Threshold speeds}

After some computations, the imaginary part of Eq. (24) at first order relative to $d$ yields the modal damping of the $n$th harmonic:

$$
d_{n}(\Omega) \approx d_{i n} \frac{\omega_{n}^{3}\left(1+\frac{d_{e n}}{d_{i n}} \Pi_{n}\right)-\left(\omega_{b n}^{2}+\frac{d_{e n}}{d_{i n}} \omega_{s n}^{2}\right) \omega_{n}+\left(\omega_{b n}^{2}-\omega_{n}^{2}\left(1+\frac{d_{e n}}{d_{i n}} \Gamma_{n}\right)\right) \Omega}{2 \omega_{n}\left(2 \Psi_{n} \omega_{n}^{2}-\omega_{s n}^{2}-\Pi_{n} \omega_{b n}^{2}\right)+\Gamma_{n}\left(\omega_{b n}^{2}-3 \omega_{n}^{2}\right) \Omega}
$$

This equation gives the stability of the system. An analytical solution for this expression is obtained when an analytical expression for $\omega_{n}$ is known. Let us assume the gyroscopic effects to be negligible, as proved to be the case in the above dimensional analysis, i.e., $\Gamma_{n} \approx 0$. Eq. (26) therefore becomes a quadratic expression which is independent of the spin speed. This directly gives the following four natural frequencies $\omega_{n p 0}$ :

$$
\omega_{n F \pm 0}=-\omega_{n B \pm 0}=\frac{1}{\sqrt{2 \Psi_{n}}} \sqrt{\omega_{s n}^{2}+\Pi_{n} \omega_{b n}^{2} \pm \sqrt{\omega_{s n}^{4}+2\left(\Pi_{n}-2 \Psi_{n}\right) \omega_{s n}^{2} \omega_{b n}^{2}+\Pi_{n}^{2} \omega_{b n}^{4}}}
$$

It should be noted that if the supports are assumed to be infinitely rigid, the above equation gives the classical relation for the lateral natural frequency of a Rayleigh-Timosheko beam with transverse shear deformation and rotatory inertia [24]

$$
\lim _{\omega_{b n} \rightarrow+\infty} \omega_{n F \pm 0}=\frac{\omega_{s n}}{\sqrt{1+\frac{I_{y}}{S}\left(\frac{n \pi}{l}\right)^{2}\left(\frac{E}{k G}+1\right)}}
$$

It should also be noted that if one sets $\Omega=0$ and $u=u_{s}$, Eq. (7) gives the same frequencies without neglecting the $\dddot{u}$ term

$$
\omega_{n 0}=\sqrt{\beta_{n}+\gamma-\sqrt{\left(\beta_{n}+\gamma\right)^{2}-4 \gamma \omega_{s n}^{2}}} \quad \text { with } \quad \beta_{n}=\frac{1}{2 \rho}\left(\frac{n \pi}{l}\right)^{2}(E+\kappa G), \gamma=\frac{\kappa G S}{2 \rho I}
$$


In the case of external and internal hysteric damping (the viscous case and the mixed case were investigated in [6]), it is therefore necessary to compute $d_{i n}$ and $d_{e n}$ carefully with the classical equivalence between viscous and hysteretic damping:

$$
c_{\mathrm{eq}}=\frac{\eta k}{|\omega|}
$$

where $c_{\text {eq }}$ is the equivalent viscous damping. Flexural critical speeds can be handled separately [20]. Equivalent external damping of the $n p$ th mode (where $p$ stands for $\{B \pm, F \pm\}$ ) can therefore be expressed as $\eta_{e} k_{e} /\left|\omega_{n p}(\Omega)\right|$, where $\omega_{n p}(\Omega)$ can be approximated by $\omega_{n p 0}$. From Eq. (25), the external modal damping can be expressed as follows:

$$
d_{e n p}=\eta_{e} \frac{\omega_{b n}^{2}}{\left|\omega_{n p 0}\right|}
$$

The internal damping has to be included in the rotating frame of reference. The excitation frequency therefore corresponds to $\left|\omega_{n p}(\Omega)-\Omega\right|$. In this case, the equivalent internal damping of the $n p$ th mode can be expressed as $\eta_{i} k_{s n} /\left(\mid \omega_{n p}(\Omega)-\right.$ $\Omega \mid l)$ where $\omega_{n p}(\Omega)$ can be approximated by $\omega_{n p 0}$. According to Eq. (25) and assuming $\Omega \geqslant 0$, the internal modal damping can be expressed as follows:

$$
d_{\text {inp }}(\Omega)=\left\{\begin{array}{cl}
\frac{\eta_{i} \omega_{s n}^{2}}{\Omega-\omega_{n p 0}} & \text { for } p \in\{B \pm\} \\
\frac{\eta_{i} \omega_{s n}^{2}}{\omega_{n p 0}-\Omega} & \text { for }\left(p \in\{F \pm\} \text { and } \Omega<\omega_{n F \pm 0}\right)
\end{array}\right.
$$

The above equivalences (39-40) and Eq. (35) can be included in Eq. (34), and the positivity of these new equations can be studied analytically. Based on the previous study [6], the following conclusion can be reached: backward whirl modes are always stable, and forward whirl modes can be unstable only in the supercritical range. This important conclusion confirms the role of hysteretic damping in rotors described by Genta [21]. In addition, an analytical instability criterion can be written in the following form:

$$
\eta_{e} k_{e} \Phi_{n}\left(\Pi_{n} \omega_{n F+0}^{2}-\omega_{s n}^{2}\right)-\eta_{i} k_{s n}\left(\omega_{n F+0}^{2}-\omega_{b n}^{2}\right) \begin{cases}<0 & \Longrightarrow \omega_{\text {th } n F+}=\omega_{n F+0} \\ \geqslant 0 & \Longrightarrow \text { stable }\end{cases}
$$

and

$$
\eta_{e} k_{e} \Phi_{n}\left(\Pi_{n} \omega_{n F-0}^{2}-\omega_{s n}^{2}\right)-\eta_{i} k_{s n}\left(\omega_{n F-0}^{2}-\omega_{b n}^{2}\right) \begin{cases}>0 & \Longrightarrow \omega_{\text {th } n F-}=\omega_{n F-0} \\ \leqslant 0 & \Longrightarrow \text { stable }\end{cases}
$$

It then suffices to compute the lowest instability speed to be able to determine the threshold speed of the shaft:

$$
\omega_{\text {th }}=\min _{n \in \mathbb{N}^{*}, p \in F \pm}\left(\omega_{\text {th } n p}\right)
$$

\subsection{Equivalent stiffness and damping of the shaft model}

Flexural vibrations of a composite tube create a tensile/compressive state in the laminate in the direction of the tube axis. The laminate equivalent modulus in this loading case can be computed via the LT as previously described in the case of a symmetrical laminate in [25] (see AppendixA):

$$
\begin{aligned}
E & =\frac{1}{t_{s} a_{11}} \\
G & =\frac{1}{t_{s} a_{33}} \\
v & =-\frac{a_{12}}{a_{11}}
\end{aligned}
$$

where $\mathbf{a}=\mathbf{A}^{-1}$. Despite this restriction, the above equations will be used even if the laminate is unsymmetrical (see 3.1.5).

The shear coefficient is approximated with the relation obtained for a cylinder consisting of a homogeneous material

$$
\kappa=\frac{2(1+v)}{4+3 v}
$$


The equivalent loss factor denoted $\eta_{i}$ is computed with Adams and Bacon's theory [1, 26, 27], using complex properties of the ply $E_{11}^{*}, E_{22}^{*}$ and $G_{12}^{*}$. In this theory, the strain energy is computed in the case of a particular plane stress state (e.g. tension/compression in the case of the shaft) with the LT. A dissipative energy is then defined for each strain energy term (longitudinal tension/compression, transverse tension/compression and in-plane shear). The internal loss factor can then be obtained directly by dividing the local dissipative energy by the local strain energy, i.e.,

$$
\eta_{i}=\frac{\sum_{k=1}^{p}\left(\frac{1}{2} \int_{h_{k-1}}^{h_{k}} \boldsymbol{\varepsilon}^{T} \boldsymbol{\eta} \boldsymbol{\sigma} \mathrm{d} z\right)}{\sum_{k=1}^{p}\left(\frac{1}{2} \int_{h_{k-1}}^{h_{k}} \boldsymbol{\varepsilon}^{T} \boldsymbol{\sigma} \mathrm{d} z\right)} \quad \text { with } \quad \boldsymbol{\eta}=\left[\begin{array}{ccc}
\eta_{11} & 0 & 0 \\
0 & \eta_{22} & 0 \\
0 & 0 & \eta_{12}
\end{array}\right]
$$

where $\boldsymbol{\eta}$ is the loss factor matrix expressed in the ply frame of reference (see Eqs. (A.2) and (A.3) for the definition of stresses and strains). In the case of unsymmetrical laminate, as it will be discussed latter in 3.1.5, the composite coupling mechanism can be neglected due to structural effects. Hence, assuming the coupling matrix $\mathbf{B}$ to be null in the computation of Eq. (48), a better approximation of internal loss factor will be obtained.

The damping properties of the ply, which are assumed to be independent of the frequency, are assumed to be equal to $\eta_{11}=0.11 \%, \eta_{22}=0.70 \%$ and $\eta_{12}=1.10 \%$ as in [19].

\section{Numerical results}

\subsection{The case of Zinberg and Symonds' shaft}

In order to assess the accuracy of the critical speed predictions, the case of the helicopter tail rotor shaft described by Zinberg and Symonds [14] is studied with the equations obtained above. The properties of boron/epoxy material are listed in Table 1 and the geometric parameters are: $l=2.47 \mathrm{~m}, t_{s}=1.321 \mathrm{~mm}$ and $r_{m}=62.84 \mathrm{~mm}$. The laminate stacking sequence is: $\left[90^{\circ}, 45^{\circ},-45^{\circ}, 0^{\circ}{ }_{6}, 90^{\circ}\right]$ from inner to outer surface. In their study, Zinberg and Symonds determined the main properties of the shaft using various experimental methods. They obtained the first natural frequency at $5500 \mathrm{rev} \mathrm{min}^{-1}$ with a shaker at the span center, which imposed a sinusoidal input. They estimated the first critical speed by performing a rotation test. Since the oscillation amplitude of the shaft was too great, the test was stopped at $5100 \mathrm{rev} \mathrm{min}^{-1}$ and only two points in the unbalanced response curve were measured at the shaft center which was $0.64 \mathrm{~mm}$ and $1.27 \mathrm{~mm}$ at $3700 \mathrm{rev} \mathrm{min}^{-1}$ and $4500 \mathrm{rev} \mathrm{min}^{-1}$, respectively. The authors extrapolated the unbalanced response with the undamped theoretical curve (without giving any details about the rotor unbalance) and predicted the critical speed to be $6000 \mathrm{rev} \mathrm{min}^{-1}$. The torsional modulus was measured by performing a pure torsion test and found to be $16.5 \mathrm{GPa}$. The longitudinal modulus was found to be $142 \mathrm{GPa}$ with a shaft tested in the same way as a cantilever beam. With this value, the first critical speed was determined with the method now known as EMBT and a value of 5840 rev min $^{-1}$ was obtained.

This long composite shaft was investigated by several authors, taking various factors into account, such as the transverse shear, the laminate couplings, etc (see. Table 2). Since we cannot be sure of the accuracy of experimental estimates of the critical speed, all the results obtained here will be compared below with the theoretical values obtained by Zinberg and Symonds. It should be noted that the value of the mean radius was confused in the original studies [7, 9, 11-13] with that of the outer radius $(0.0635 \mathrm{~m})$. In comparison with the original data, all these critical speeds have been corrected in Table 2 by simply multiplying them by the ratio between the two radii (i.e., $0.06284 / 0.0635$ ). This ratio gives an approximation of the differences in the value of $\sqrt{I_{x} / S}$.

Most of these authors obtained a critical speed showing good agreement with the experimental values. Using Donnel's theory, dos Reis et al. [7] underestimated the critical speed, whereas Kim and Bert [8] overestimated this speed using the same theory. Kim and Bert mentioned that they previously established that Donnel's theory is not suitable for use with long beams.

To determine the influence of various factors on the shaft critical speed, the classical relation $\omega_{s 1}$ in Eq. (25) was used as the basis of our calculations. This equation gives almost the same critical speed as that obtained by Zinberg and Symonds [14] with an error of only $0.2 \%$, which was certainly a round-off error.

\subsubsection{Effects of the rotatory inertia}

The rotatory inertia was the first parameter to be investigated. The comparisons made in Table 2 between Eq. (25) and Eq (36), where $\frac{E}{K G}$ is assumed to be null, show that this factor was responsible for a decrease of only $0.1 \%$ in the critical speed predictions.

\subsubsection{Effects of the gyroscopic forces}

The Campbell diagram shows the evidence that the gyroscopic effects are small in the first mode (see. Fig. 2). It can be seen from Eqs. (31) and (33) that the first critical speed corresponds to the backward mode. The effects of the gyroscopic 
Table 2: First critical speed in Zinberg and Symonds' case [14] using various theories (Diff. = relative difference in comparison with classical EMBT, Gyr. = gyroscopic effect, R.I. = rotatory inertia, Sh. $=$ transverse shear effect, Sup. $=$ support stiffness effect, Co. $=$ laminate coupling mechanism and E.F. $=$ end fitting)

\begin{tabular}{|c|c|c|c|c|c|c|c|c|c|}
\hline Investigators & $\begin{array}{l}\text { Critical sp. } \\
\left(\text { rev } \min ^{-1}\right)\end{array}$ & Diff. (\%) & Gyr. & R.I. & Sh. & Sup. & Co. & E.F. & Theory \\
\hline \multirow[t]{2}{*}{ Zinberg et al. [14] } & 5840 & 0 & & & & & & & EMBT \\
\hline & 5500 & -5.8 & & & & & & & Experimental without rotation \\
\hline Dos Reis et al. [7] & $4899 *$ & -16.1 & & & $\bullet$ & $\bullet \star$ & $\bullet$ & & $\begin{array}{l}\text { FE beam with stiffnesses determined } \\
\text { using Donell's shell theory }\end{array}$ \\
\hline \multirow{5}{*}{ Kim et al. [8] } & 5872 & 0.5 & $\bullet$ & $\bullet$ & $\bullet$ & & $\bullet$ & & Sanders' shell theory \\
\hline & 5892 & 0.9 & $\bullet$ & $\bullet$ & $\bullet$ & & $\bullet$ & & Love's first approximation shell theory \\
\hline & 5856 & 0.3 & $\bullet$ & $\bullet$ & $\bullet$ & & $\bullet$ & & Loo's shell theory \\
\hline & 5878 & 0.7 & $\bullet$ & $\bullet$ & $\bullet$ & & $\bullet$ & & Morley's shell theory \\
\hline & 6399 & 9.6 & $\bullet$ & $\bullet$ & $\bullet$ & & $\bullet$ & & Donnel's shell theory \\
\hline Gubran et al. [12] & $5494 *$ & -5.9 & $\bullet$ & $\bullet$ & $\bullet$ & & $\bullet$ & & MEBT including bending-twisting effects \\
\hline \multirow[t]{2}{*}{ Sino et al. [13] } & $5707 *$ & -2.3 & $\bullet$ & $\bullet$ & & $\bullet \triangleright$ & & & SHBT (FE beam) \\
\hline & $5378^{*}$ & -7.9 & $\bullet$ & $\bullet$ & $\bullet$ & $\bullet$ & & & SHBT (FE beam) \\
\hline \multicolumn{10}{|l|}{ Present study } \\
\hline$\omega_{s 1}$ in Eq. (25) & 5852 & 0.2 & & & & & & & EMBT \\
\hline Eq. (36) with $\frac{E}{k G}=0$ & 5843 & 0.1 & & $\bullet$ & & & & & EMBT \\
\hline Eq. (33) with $\frac{E}{k G}=0$ & 5825 & -0.3 & $\bullet$ & $\bullet$ & & & & & EMBT \\
\hline Eq. (37) & 5697 & -2.5 & & $\bullet$ & $\bullet$ & & & & EMBT \\
\hline Eq. (36) & 5696 & -2.5 & & $\bullet$ & $\bullet \dagger$ & & & & EMBT \\
\hline Eq. (33) & 5679 & -2.8 & $\bullet$ & $\bullet$ & $\bullet \dagger+$ & & & & EMBT \\
\hline ABAQUS with & 5622 & -3.7 & & $\bullet$ & $\bullet$ & & $\bullet$ & $\bullet$ & Thin shell FE (S4R) / \\
\hline realistic end fit. & & & & & & & & & 3D FE (C3D8R) for end fittings \\
\hline
\end{tabular}

* corrected values due to the error in the mean tube radius value $-\dagger \dddot{u}$ term neglected $-\ddagger \dddot{u}$ term neglected -

${ }^{\star} k_{e}=1.74 \times 10^{12} \mathrm{~N} \mathrm{~m}^{-1}-{ }^{\diamond}$ exist in the reference study but not used here $-{ }^{\circ} k_{e}=1 \times 10^{7} \mathrm{~N} \mathrm{~m}^{-1}$ and $m_{e}=0.48 \mathrm{~kg}$

forces can be obtained by comparing the values obtained with Eqs (36) and (33), assuming the term $\frac{E}{K G}$ to be null in both cases. This comparison shows that the gyroscopic effects were responsible for a decrease of only $0.4 \%$. This result confirms our previous suggestion that experimental values of the critical speed should be equal to approximately the first natural experimental frequency, i.e., $5500 \mathrm{rev}^{-1} \mathrm{~m}^{-1}$.

\subsubsection{Effects of the transverse shear}

All the authors quoted above except for Zinberg and Symonds included the transverse shear effects. The effects of this factor can be determined by comparing the values obtained with $\mathrm{Eq}$ (36), where $\frac{E}{K G}$ is assumed to be null, and Eq. (37). These comparisons showed that the transverse shear effect decreased the critical speed by about $2.6 \%$. A similar difference was obtained whether or not the term $\frac{E}{K G}$ was removed from Eq. (33), Eq. (35) or Eq. (28) (see Table 2). Sino et al. [13] obtained a value of about 5.6\%. Comparisons between the values obtained with Eqs (37) and (36) also showed that the effects of $\dddot{u}$ term are negligible. Comparisons between the values obtained with Eqs (36) and (33) confirmed in addition that the term $\dddot{u}$ is negligible if the gyroscopic effects are assumed to be equal to a value of about $0.4 \%$. 


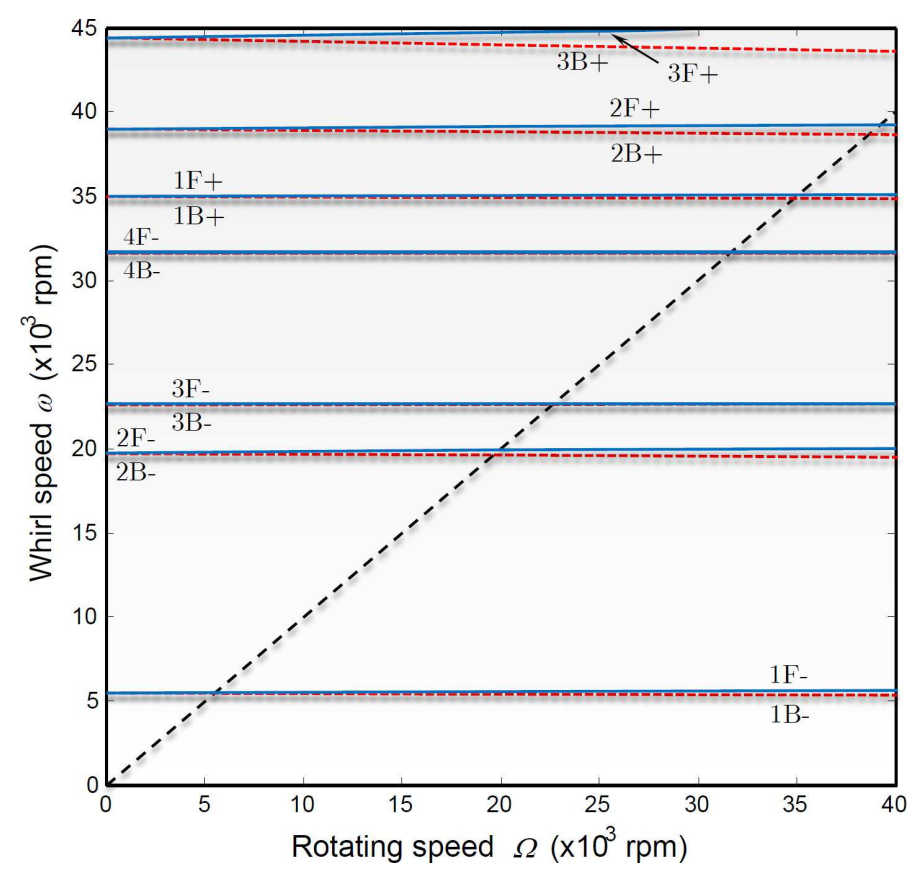

Figure 2: The Campbell diagram of the Zinberg and Symonds' shaft (with $k_{e}=10^{7} \mathrm{~N} \mathrm{~m}^{-1}$, (-) forward modes, (-- -) backward modes).

Table 3: Normalized middle plane strains, curvatures and elastic strain energy in Zinberg and Symonds composite for the first mode

\begin{tabular}{|c|c|c|c|c|c|c|c|}
\hline Method & $\varepsilon_{x x}^{0}$ or $\varepsilon_{r r}^{0}$ & $\varepsilon_{y y}^{0}$ or $\varepsilon_{\theta \theta}^{0}$ & $\varepsilon_{x y}^{0}$ or $\varepsilon_{\theta \theta}^{0}$ & $k_{x x}$ or $k_{r r}\left(\mathrm{~m}^{-1}\right)$ & $k_{y y}$ or $k_{\theta \theta}\left(\mathrm{m}^{-1}\right)$ & $k_{x y}$ or $k_{r \theta}\left(\mathrm{m}^{-1}\right)$ & $\widehat{U_{e l}}\left(\mathrm{~N} \mathrm{~m}^{-1}\right)$ \\
\hline FEM Plate (static tension) & 1 & -0.291 & 0.0555 & -1007 & 271 & 360 & 0.4027 \\
\hline Laminate theory (static tension) & 1 & -0.294 & 0.0680 & -1037 & 299 & 415 & 0.4026 \\
\hline Laminate theory with $\mathbf{B}=\mathbf{0}$ (static tension) & 1 & -0.258 & 0 & 0 & 0 & 0 & 0.4452 \\
\hline
\end{tabular}

\subsubsection{Effects of the support stiffness}

The effects of the support stiffness were investigated using Eqs (35) and (28). In the reference study, no information was provided about the stiffness of the test rig. The bearing and the test rig were assumed here to be very stiff. The stiffness was therefore taken to be equal to $10^{7} \mathrm{~N} \mathrm{~m}^{-1}$. The mass of the rotating part was estimated at $0.48 \mathrm{~kg}$ with the CAD drawing. With these numerical values, the critical speed obtained was only approximately $2 \%$ below the reference value. This difference is of the same order of magnitude as with the effects of the transverse shear.

\subsubsection{Effects of the composite coupling}

The coupling mechanisms in the laminate were investigated with the FE method in ABAQUS and compared with the results obtained using the LT (see AppendixA). The finite elements (S4R) were of the thin shell type. First of all, a plate measuring $0.1 \mathrm{~m}$ in the $\mathbf{x}$ direction by $0.06 \mathrm{~m}$ in the $\mathbf{y}$ direction was studied in order to test the validity of the comparisons between FE method and LT. This plate consisted of the same laminate as Zinberg and Symonds' shaft. A climb condition was applied to one of the smaller edges, and tension was applied to the opposite edge. The strain field $\left(\varepsilon_{x x}, \varepsilon_{y y}\right.$ and $\left.\varepsilon_{x y}\right)$ was analyzed at the plate center. As it can be seen in Eq. (A.2), the linear evolution of the strains is given by the middle plane strains (denoted $\varepsilon_{x x}^{0}, \varepsilon_{y y}^{0}$ and $\varepsilon_{x y}^{0}$ ) and the curvatures (denoted $k_{x x}, k_{y y}$ and $k_{x y}$ ). Fig 3 a shows the strain field obtained both in the laminate frame of reference with the FE method and directly with the LT by applying a normal force. To compare the results obtained with the two methods, the middle plane longitudinal strain $\varepsilon_{x x}^{0}$ was normalized to 1 . In this figure, it is worth noting that the strains in each of the plies are a function of the height, although this is a tension loading case. This height-dependence was due to unsymmetry of the laminate i.e. to the occurrence of bending-stretching coupling and bending-twisting coupling. The excellent agreement observed with the LT shows that the FE method can be used to understand what happens in the case of unsymmetrical composite cylinders. The comparisons between middle plane strains, curvatures and elastic strain energies made in Table 3 also confirm the existence of good agreement between the two methods, where the elastic strain energy per surface unit can be expressed as follows:

$$
\widehat{U_{\mathrm{el}}}=\frac{1}{2} \sum_{k=1}^{p} \int_{h_{k-1}}^{h_{k}} \sigma_{x x} \varepsilon_{x x}+\sigma_{y y} \varepsilon_{y y}+\sigma_{x y} \varepsilon_{x y} \mathrm{~d} z
$$




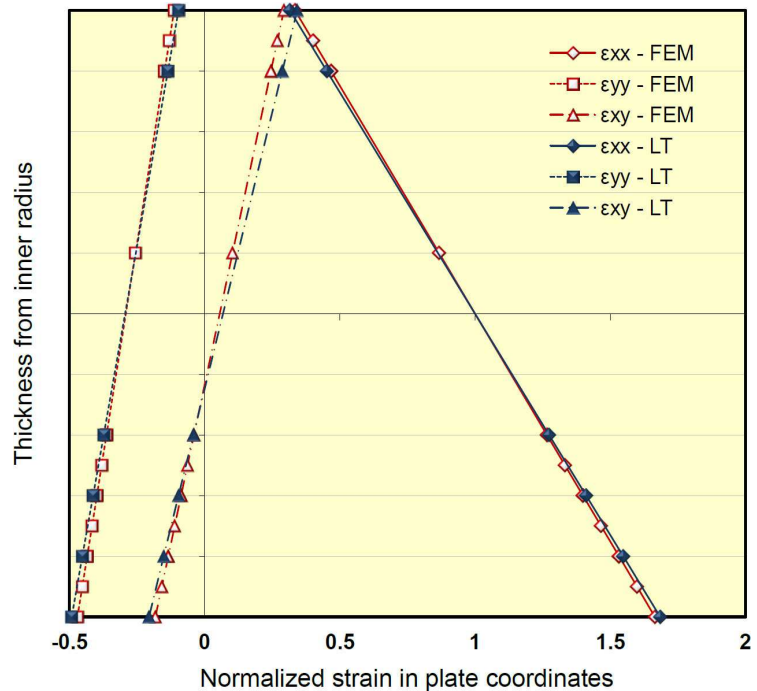

(a)

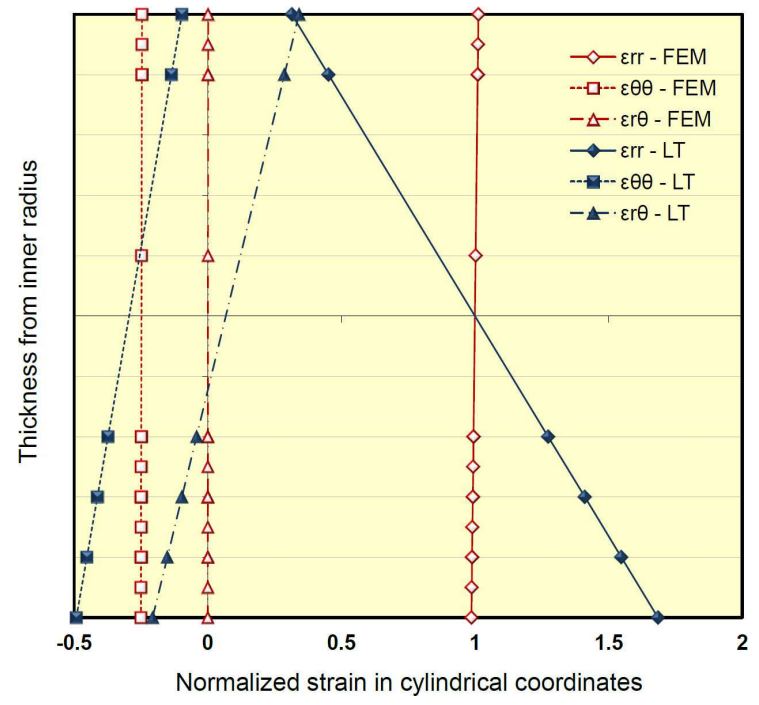

(b)

Figure 3: Normalized strain distribution in the first mode of a composite with Zinberg and Symonds composite. (a) ABAQUS FEM plate vs. laminate theory; (b) ABAQUS FEM cylindrical shell vs. laminate theory.

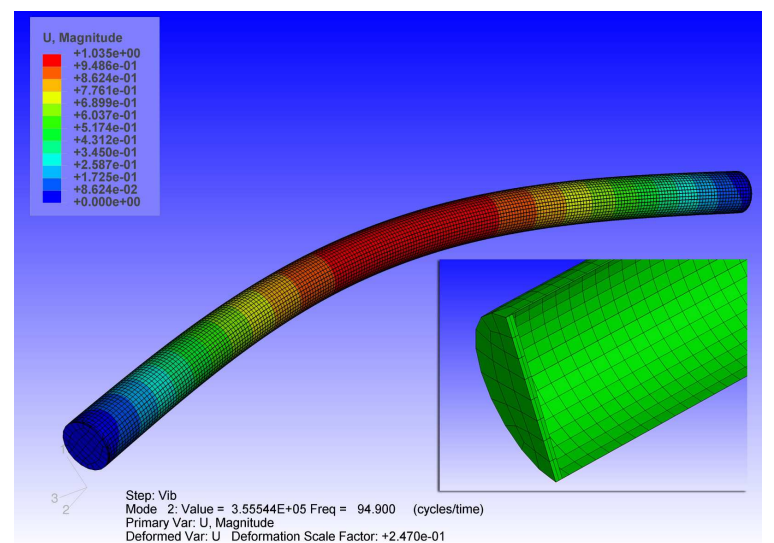

(a)

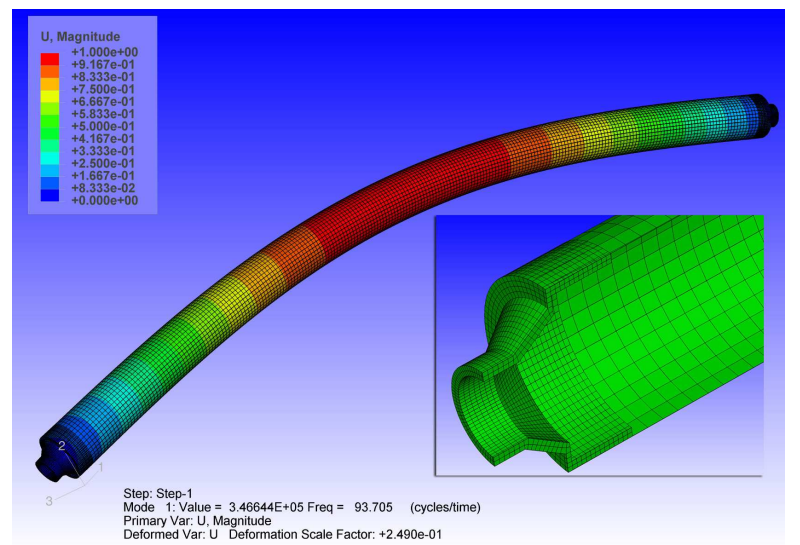

(b)

Figure 4: Finite element simulation of the Zinberg and Symonds [14] shaft with ABAQUS: effects of the end fittings on the first natural frequency. (a) First mode of the shaft with thin and infinitely rigid end fittings and detailed mesh of an end fitting; (b) first mode of the shaft with elastic end fittings and detailed mesh of an end fitting.

Zinberg and Symonds' shaft was studied in the first bending mode without any rotating effects. The shaft was closed with thin end fittings assumed to be infinitely rigid and quasi massless (Fig. 4a), which were meshed using linear hexahedral elements of the C3D8R type. Pinned and slider conditions were applied at the center of these end fittings. Fig 3b compares the normalized strain field obtained in the laminate frame of reference between FE method and LT. On contrary to the case of plates, no agreement was found to exist between the results obtained with the two methods. A structural effect occurred in this case, which blocked all the curvatures. Only the $k_{r r}$ curvature can be distinguished in the figure, but it is very small in comparison with that observed in the case of plates (see Table 3). This results in a completely different stress field, which makes it possible to cancel the curvatures due to the coupling effects. If this field is now compared with a modified LT where the coupling matrix B (defined by the Eqs (A.5-A.6)) is taken to be null, good agreement is observed with the FE results (Fig 5a). With this assumption, Fig 5b and Table 3 also show the existence of excellent agreement in terms of the stress field and the elastic strain energy. It is concluded that this simplified approach accurately predicts the eigenvector in the first bending mode. There is practically no elastic strain energy in the curvatures, and the eigenvalue is practically not affected by the coupling mechanism due to the tubular structure. A good estimate of the critical speed can therefore probably be obtained by neglecting the coupling mechanism, and Eqs. (44-46) can be used even if the laminate is unsymmetrical.

\subsubsection{Effects of the end fittings}

The effects of the end fittings are the last effects studied with the FE method. Since no information is available about the exact geometry of the original end fittings, the FE model presented in Fig. $4 \mathrm{~b}$ is based on the picture published in Zinberg 


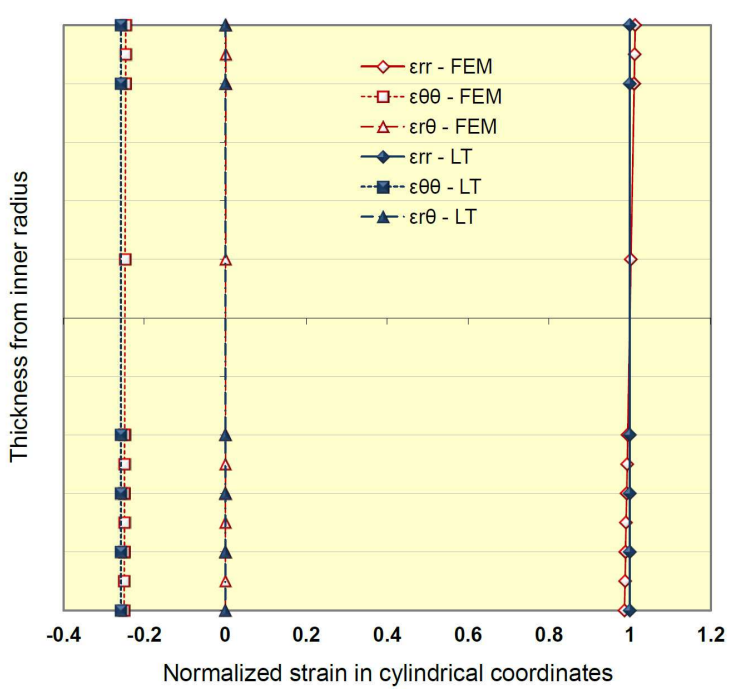

(a)

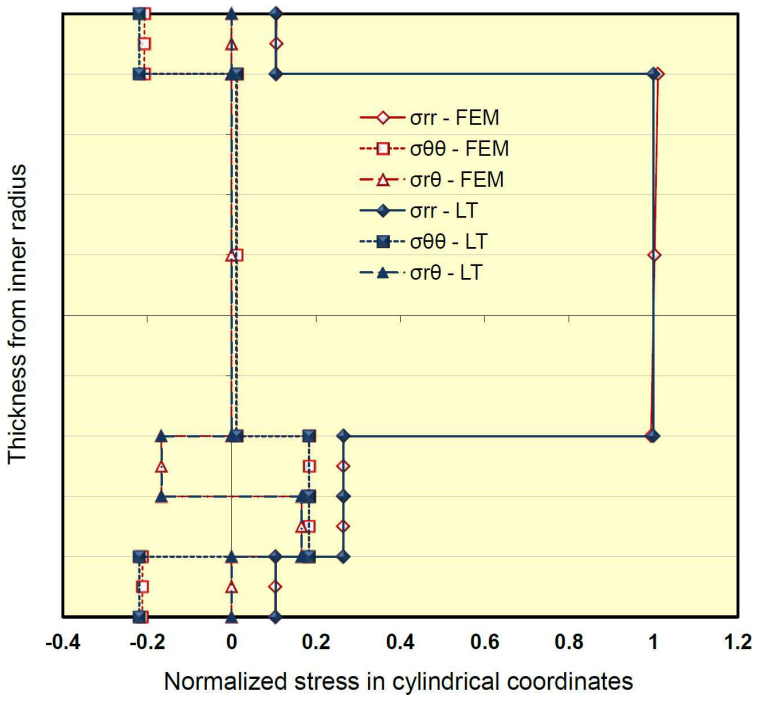

(b)

Figure 5: Normalized strain distribution (a) and stress distribution (b) in the first Zinberg and Symonds' shaft mode: ABAQUS shell vs. laminate theory with $\mathbf{B}=\mathbf{0}$.

and Symonds' paper. A smaller tube is assumed to maintain the total shaft length between boundary conditions at a value of $2.47 \mathrm{~m}$. The end fittings are assumed to be made of aluminum. As in the previous case, the end fittings are meshed using linear hexahedral elements of the C3D8R type. Tie conditions are applied between the junction and the composite tube. The computations show that these end fittings decrease the critical speed by $1.3 \%$, which is relatively little (see Table 2).

\subsubsection{Partial conclusion on the equations considered}

It can be concluded that the first critical speed of a composite shaft can be accurately predicted with Eqs. (31-33), which takes the rotatory inertia, gyroscopic effect and transverse shear effect into account, assuming the composite coupling to be canceled out by structural effects. A more accurate estimate of the critical speed can be obtained with Eqs. (27-28) if the stiffnesses and masses of the supports are known.

\subsection{Dynamic behavior and stability of a composite shaft with internal hysteretic damping}

No numerical studies on damping instabilities in a single composite shaft have been published so far to the best of our knowledge. Only Sino et al. [13] have investigated the case of a composite shaft with two disks. In the present study, comparisons were made between the results obtained with our theory and EMBT in the case of six different composite stacking sequences (Table 4). The stacking sequences used for this purpose, whether or not they were symmetrical and of the same thickness, corresponded to classical configurations previously studied in the literature, most of which resist torque loads and/or maximize the longitudinal stiffness [28]. Since EMBT and ECMBT do not take the order of plies into account, only one order of plies was studied for each laminate stacking sequence. The properties of T300/5208 carbon/epoxy material are listed in Table 1, and the geometric parameters of the shaft studied were: $l=2 \mathrm{~m}, t_{s}=1.5 \mathrm{~mm}$ and $r_{m}=39.25 \mathrm{~mm}$. To ensure stability in the supercritical regime, the shaft was mounted on dissipative supports with a low stiffness $k_{e}=2 \times 10^{6} \mathrm{~N} \mathrm{~m}^{-1}$ and a large loss factor $\eta_{e}=7 \%$. The bearing mass on each side $\left(m_{b}\right)$ was equal to $1 \mathrm{~kg}$.

In this example, the rigid-body modes obtained were mostly in between the first and second flexural modes obtained with EMBT (see Table 4). This amplified the coupling mechanism between the two types of modes. EMBT does not take the rigid-body modes into account, which can result in large errors in this case. With ECMBT, four critical speeds (two backward modes and two forward modes) were determined for each flexural mode. For example, in the first mode of shaft $\mathrm{N}^{\circ} 1, \omega_{c 1 F+}$ and $\omega_{c 1 F-}$ correspond to two coupled modes. The first mode is mainly the cylindrical mode and the second one is mainly the first flexural mode. Frequencies are shifted from $177 \mathrm{~Hz}$ to $224 \mathrm{~Hz}$ and $102 \mathrm{~Hz}$ to $95 \mathrm{~Hz}$, respectively. A similar pattern is observed in the backward modes. It is amplified when the flexural frequency is very similar to the rigid-body frequency. For example, the second mode of the shaft $\mathrm{N}^{\circ} 3$ at $168 \mathrm{~Hz}$ and the cylindrical mode at $177 \mathrm{~Hz}$ became two coupling modes at $157 \mathrm{~Hz}$ and $232 \mathrm{~Hz}$, respectively. It should be noted that differences were also found to exist between the EMBT and ECMBT data obtained due to the gyroscopic effect, the rotatory inertia and the transverse shear. In particular, the gyroscopic effect can be clearly distinguished in the second modes from the differences between backward and forward frequencies (e.g. $\omega_{c 2 F+}$ and $\omega_{c 2 B+}$ of the shaft $\left.\mathrm{N}^{\circ} 1\right)$. As regards the stability, the shafts with the lowest longitudinal stiffness and the highest longitudinal damping (shafts $\mathrm{N}^{\circ} 2$ and 3 ) were already unstable after the first flexural mode. These configurations, involving 
Table 4: Comparison between ECMBT and EMBT with various stacking sequences in a composite shaft (T300/5208) mounted on dissipative supports $\left(l=2 \mathrm{~m}, t_{s}=1.5 \mathrm{~mm}, r_{m}=39.25 \mathrm{~mm}, k_{e}=2 \times 10^{6} \mathrm{~N} \mathrm{~m}^{-1}, \eta_{e}=7 \%\right.$ and $\left.m_{b}=1 \mathrm{~kg}\right)$

\begin{tabular}{|c|c|c|c|c|c|c|c|c|c|}
\hline Shaft & & $\mathrm{N}^{\circ}$ & & 1 & 2 & 3 & 4 & 5 & 6 \\
\hline \multirow[t]{3}{*}{ Stacking sequence } & & & & {$[ \pm 15]_{3 s}$} & {$[ \pm 30]_{3 s}$} & {$[ \pm 45]_{3 s}$} & {$\left[90,0_{3}\right.$} & {$\left[90_{2}, 0_{7}\right.$} & {$\left[90_{2}, 0_{8}\right.$,} \\
\hline & & $G$ & $\mathrm{GPa}$ & 17.0 & 36.7 & 46.6 & 20.3 & 12.6 & 13.7 \\
\hline & & $v$ & - & 1.060 & 1.370 & 0.747 & 0.295 & 0.101 & 0.203 \\
\hline \multirow[t]{2}{*}{ Rigid-body modes $^{a}$} & cylindrical & $\omega_{b 1}$ & $\mathrm{~Hz}$ & \multicolumn{6}{|c|}{176.8} \\
\hline & conical & $\omega_{b 2}$ & $\mathrm{~Hz}$ & \multicolumn{6}{|c|}{204.9} \\
\hline \multirow[t]{2}{*}{ 1st flexural modes } & EMBT & $\omega_{s 1}$ & $\mathrm{~Hz}$ & 101.9 & 67.7 & 42.1 & 86.9 & 89.7 & 96.1 \\
\hline & ECMBT & $\omega_{c 1 F+}$ & $\mathrm{Hz}$ & 224.3 & 218.0 & 214.8 & 221.0 & 220.7 & 222.2 \\
\hline \multirow[t]{5}{*}{ 2nd flexural modes } & EMBT & $\omega_{s 2}$ & $\mathrm{~Hz}$ & 407.7 & 270.8 & 168.4 & 347.6 & 358.9 & 384.2 \\
\hline & ECMBT & $\omega_{c 2 F+}$ & $\mathrm{Hz}$ & 414.3 & 299.3 & 232.0 & 363.4 & 363.6 & 387.0 \\
\hline & & $\omega_{c 2 F-}$ & $\mathrm{Hz}$ & 201.4 & 194.2 & 157.1 & 199.6 & 199.9 & 200.8 \\
\hline & & $\omega_{c 2 B-}$ & $\mathrm{Hz}$ & 201.3 & 194.0 & 155.5 & 199.6 & 199.9 & 200.7 \\
\hline & & $\omega_{c 2 B+}$ & $\mathrm{Hz}$ & 408.2 & 294.8 & 230.5 & 358.0 & 358.5 & 381.5 \\
\hline Threshold speed & ECMBT & $\omega_{\text {th }}$ & $\mathrm{Hz}$ & 824.4 & 65.8 & 41.6 & 729.3 & 713.0 & 760.6 \\
\hline
\end{tabular}

ply orientations of $\pm 45^{\circ}$ or $\pm 30^{\circ}$, are not suitable for use in the supercritical regime. The other four stacking sequences can be used in the supercritical regime and are stable until the rotational speed reaches the third mode.

The second study dealt with the effects of the shaft length on the dynamic stability. Shaft $\mathrm{N}^{\circ} 4$ was studied with variable lengths in the 0.8 to 2.5 meter range (Fig. 6a). The figure shows the decrease in the critical speeds which occurred with increasing shaft lengths. The coupling between the flexural and rigid-body modes was found to occur at around $1.3 \mathrm{~m}$. In particular, at $1.36 \mathrm{~m}$, where $\omega_{b 1}=\omega_{s 1}=188 \mathrm{~Hz}$, the frequencies $\omega_{c 1 F+}$ and $\omega_{c 1 F-}$ differed considerably from this frequency. The stability domain showed a sawtooth pattern. Up to $0.85 \mathrm{~m}$, the shaft cannot be supercritical. Between 0.85 and $1.3 \mathrm{~m}$, the instability occurred after the second flexural mode. From 1.3 to $2.3 \mathrm{~m}$, the instability shifted to the third flexural mode. Beyond $2.3 \mathrm{~m}$, it developed after the fourth flexural mode. It is sometimes possible to increase the stability by increasing the shaft length, as shown by the mathematical form of the stability criteria (Eqs. (41-42)).

As can be seen in these criteria, the support stiffness can be used as an optimized variable to increase the stability margins, as shown in Fig 6b. The same support material was again used for this purpose, but the theoretical size of the support was reduced in order to increase the stiffness from $10^{5}$ to $10^{8} \mathrm{~N} \mathrm{~m}^{-1}$. The external loss factor remained unchanged in this case. It can be seen from this figure that the stability domain increased when the first flexural frequency was near the cylindrical rigid-body frequency. This can be explained by the fact that the energy dissipation necessitates the greatest displacement of the supports. This is only possible when the rigid-body modes are excited with flexural modes, i.e., when both frequencies are almost similar.

\section{Conclusion}

In conclusion, the critical speeds of a composite shaft mounted on viscoelastic supports were obtained here in the analytic form, adopting Timoshenko assumptions and including the gyroscopic effects. This method, which we have called ECMBT, accurately predicts the first critical speed of Zinberg and Symonds' composite shaft. In fact, in the case of this example, it was established here numerically that the rotatory inertia and the gyroscopic effect are negligible because they affect the first critical speed prediction by only $0.1 \%$ and $0.4 \%$, respectively. Likewise, the effects of the transverse shear amounted to only about $2.6 \%$, probably due to the slenderness of the shaft. FE simulations showed that the tubular structure, unlike that of plates, blocks the bending-stretching coupling and bending-twisting coupling mechanisms in the unsymmetrical laminate. These factors therefore have little effect on the critical speed. The effects of the end fittings were investigated with the FE method and also found to be small. One of the main advantages of the ECMBT method is that it takes the stiffness of the 


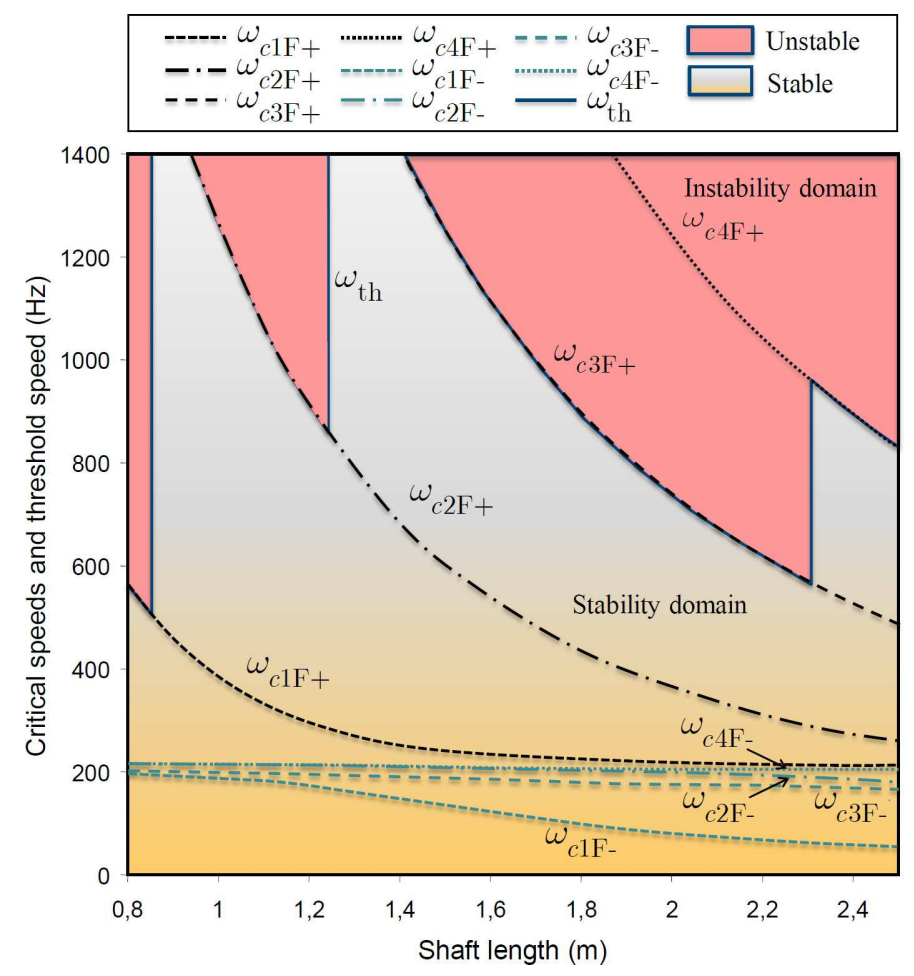

(a)

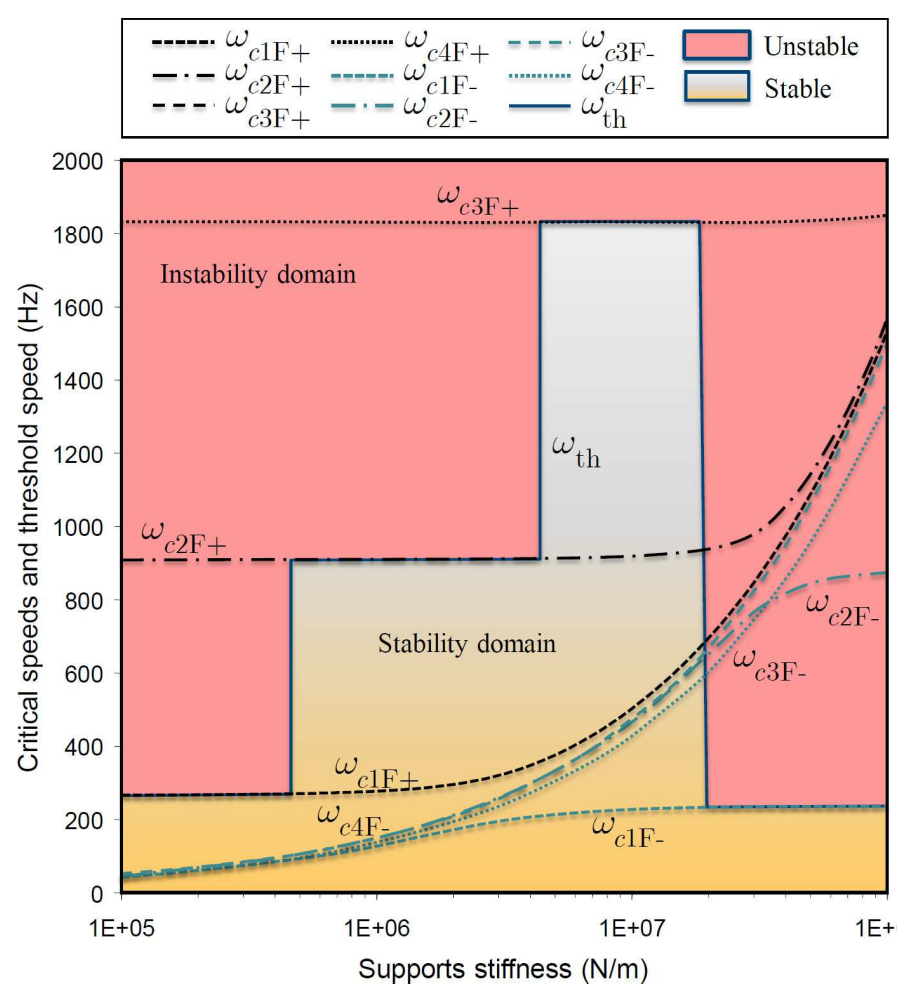

(b)

Figure 6: Evolution of the critical speeds and threshold speed of a composite shaft (T300/5208) mounted on dissipative supports $\left(\left[90,0_{3}, 45,-45\right]_{s}\right.$, $t_{s}=1.5 \mathrm{~mm}, r_{m}=39.25 \mathrm{~mm}, \eta_{e}=7 \%$ and $m_{b}=1 \mathrm{~kg}$ ). (a) Critical speeds and threshold speed vs. the shaft length with $k_{e}=2 \times 10^{6} \mathrm{~N} \mathrm{~m}^{-1} ;(\mathrm{b}) \mathrm{critical}$ speeds and threshold speed vs. the support stiffness with $l=1.2 \mathrm{~m}$.

shaft support into account. This factor can be of the same order of magnitude as the transverse shear effect in the case of rigid supports. It cannot be neglected in the case of flexible supports.

Since the damping which occurs in carbon/epoxy material resembles the hysteretic damping model much more closely than viscous damping, shaft instabilities due to rotating damping were investigated here with the hysteretic damping model. With the equivalent viscous damping approach, the threshold speed can be obtained in ECMBT using an analytic criterion. A study on various composite shafts showed that the internal damping significantly affects the dynamic stability. In particular, shafts consisting of $\pm 45^{\circ}$ or $\pm 30^{\circ}$ plies are generally not suitable for use in the supercritical regime due to their very high loss factors, which amounted here to up to $2.0 \%$ and $1.2 \%$, respectively. All the configurations including $0^{\circ}$ plies tested were found to be less dissipative and to be stable up to the third flexural critical speed. Lastly, a parametric study on the effects of the shaft length and the support stiffness on the stability of composite shafts showed the importance of the support stiffness. In particular, the stability of the shaft increases greatly when the first flexural frequency is approximately equal to the cylindrical rigid-body frequency.

\section{AppendixA. Some results of the laminate theory (LT)}

We considered a composite plate with a small thickness constituted of $p$ plies and perpendicular to the $\mathbf{z}$ direction. In the case of the Kirchoff-Love theory, a segment orthogonal to the middle plane remains straight and orthogonal to the middle plane after deformation. With these assumptions, the displacement field $\mathbf{u}$ can be written in the following simplified form

$$
u_{x}(\mathbf{x})=u_{x}^{0}(x, y)+z \frac{\partial u_{z}}{\partial x}(x, y) \quad ; \quad u_{y}(\mathbf{x})=u_{y}^{0}(x, y)+z \frac{\partial u_{z}}{\partial y}(x, y) \quad ; \quad u_{z}(\mathbf{x})=u_{z}^{0}(x, y)
$$

where $\mathbf{u}^{0}$ is the displacement field of the middle plane. The strain in the plate can be deduced from the previous field

$$
\boldsymbol{\varepsilon}(\mathbf{x})=\boldsymbol{\varepsilon}^{0}(x, y)+z \mathbf{k}(x, y) \quad \text { with } \quad \boldsymbol{\varepsilon}^{T}=\left[\begin{array}{lll}
\varepsilon_{x x} & \varepsilon_{y y} & \varepsilon_{x y}
\end{array}\right] ; \mathbf{k}^{T}=\left[\begin{array}{lll}
k_{x x} & k_{y y} & k_{x y}
\end{array}\right]
$$

where $\varepsilon^{0}$ and $\mathbf{k}$ are the strain field of the middle plane and the curvature of the middle plane, respectively. In the orthotropic frame of reference $(\mathbf{1}, \mathbf{2}, \mathbf{3})$ (where $\mathbf{1}$ and $\mathbf{2}$ correspond to the fiber and the transverse directions, respectively), the in-plane 
orthotropic elastic law is

$$
\boldsymbol{\sigma}=\mathbf{Q} \boldsymbol{\varepsilon} \quad \text { with } \quad \boldsymbol{\sigma}^{T}=\left[\begin{array}{lll}
\sigma_{x x} & \sigma_{y y} & \sigma_{x y}
\end{array}\right] ; \mathbf{Q}^{-1}=\left[\begin{array}{ccc}
\frac{1}{E_{1}} & -\frac{v_{12}}{E_{1}} & 0 \\
-\frac{v_{12}}{E_{1}} & \frac{1}{E_{2}} & 0 \\
0 & 0 & \frac{1}{G_{12}}
\end{array}\right]
$$

where $\mathbf{Q}$ is the stiffness matrix. The forces and the moments in the plate are obtained through the integration of the stresses along the ply thickness

$$
\mathbf{N}=\int_{-\frac{h}{2}}^{\frac{h}{2}} \sigma \mathrm{d} z ; \mathbf{M}=\int_{-\frac{h}{2}}^{\frac{h}{2}} \sigma z \mathrm{~d} z \quad \text { with } \quad \mathbf{N}^{T}=\left[\begin{array}{lll}
N_{x} & N_{y} & N_{x y}
\end{array}\right] ; \mathbf{M}^{T}=\left[\begin{array}{lll}
M_{x} & M_{y} & M_{x y}
\end{array}\right]
$$

which can be expressed in terms of strain via the orthotropic elastic law

$$
\left[\begin{array}{l}
\mathbf{N} \\
\mathbf{M}
\end{array}\right]=\left[\begin{array}{ll}
\mathbf{A} & \mathbf{B} \\
\mathbf{B} & \mathbf{D}
\end{array}\right]\left[\begin{array}{c}
\boldsymbol{\varepsilon}^{0} \\
\mathbf{k}
\end{array}\right]
$$

with

$$
\mathbf{A}=\frac{1}{2} \sum_{k=1}^{p} \int_{h_{k-1}}^{h_{k}} \mathbf{Q}_{k} \mathrm{~d} z ; \mathbf{B}=\frac{1}{2} \sum_{k=1}^{p} \int_{h_{k-1}}^{h_{k}} z \mathbf{Q}_{k} \mathrm{~d} z ; \mathbf{D}=\frac{1}{2} \sum_{k=1}^{p} \int_{h_{k-1}}^{h_{k}} z^{2} \mathbf{Q}_{k} \mathrm{~d} z
$$

where $\mathbf{Q}_{k}$ is the stiffness matrix of the ply $k$ expressed in the plate frame of reference $(\mathbf{x}, \mathbf{y}, \mathbf{z})$.

[1] R. D. Adams, Engineering Material Handbook, vol. 1, chap. Damping properties analysis of composites, ASME, $206-217,1987$.

[2] F. Sorge, Damping of rotor conical whirl by asymmetric dry friction suspension, Journal of Sound and Vibration 321 (1) (2009) $79-103$.

[3] F. Sorge, M. Cammalleri, On the beneficial effect of rotor suspension anisotropy on viscous-dry hysteretic instability, Meccanica (2012) 1-18.

[4] J. K. Dutt, B. C. Nakra, Stability of rotor systems with viscoelastic supports, Journal of Sound and Vibration 153 (1) (1992) $89-96$.

[5] J. K. Dutt, B. C. Nakra, Stability characteristics of rotating systems with journal bearings on viscoelastic support, Mechanism and Machine Theory 31 (6) (1996) 771-779.

[6] O. Montagnier, C. Hochard, Dynamic instability of supercritical driveshafts mounted on dissipative supports - effect of viscous and hysteretic internal damping, Journal of Sound and Vibration 305 (2007) 378-400.

[7] H. L. M. dos Reis, R. B. Goldman, P. H. Verstrate, Thin-Walled Laminated Composite Cylindrical Tubes. III. Critical Speed Analysis, ASTM Journal of Composite Technology and Research 9 (2) (1987) 58-62.

[8] C. D. Kim, C. W. Bert, Critical speed analysis of laminated composite, hollow drive shafts, Composites engineering 3 (7-8) (1993) 633-643, ISSN 0961-9526.

[9] C. W. Bert, C. K. Kim, Whirling of composite material driveshafts including bending-twisting coupling and transverse shear deformation, Journal of Vibration and Acoustics 117 (1) (1995) 7-21.

[10] S. P. Singh, K. Gupta, Composite shaft rotordynamic analysis using a layerwise theory, Journal of Sound and Vibration 191 (5) (1996) $739-756$.

[11] M. Chang, J. Chen, C. Chang, A simple spinning laminated composite shaft model, International journal of solids and structures 41 (3) (2004) $637-662$.

[12] H. B. H. Gubran, K. Gupta, The effect of stacking sequence and coupling mechanisms on the natural frequencies of composite shaft, Journal of Sound and Vibration 282 (1-2) (2005) 231-248.

[13] R. Sino, T. N. Baranger, E. Chatelet, G. Jacquet, Dynamic analysis of a rotating composite shaft, Composites Science and Technology 68 (2) (2008) 337-345.

[14] H. Zinberg, M. F. Symonds, The Development of an Advanced Composite Tail Rotor Driveshaft, in: Proceedings of the 26th Annual Forum of the American helicopter Society, Washington, United States, 1970.

[15] J. W. Lim, M. S. Darlow, Optimal sizing of composite power transmission shafting, Journal of the American Helicopters society 31 (1986) $75-83$.

[16] M. S. Darlow, J. Creonte, Optimal design of composite helicopter power transmission shafts with axially varying fiber lay-up, Journal of the American Helicopters society 40 (2) (1995) 50-56.

[17] J. Martínez-Casas, J. Fayos, F. Denia, L. Baeza, Dynamics of damped rotating solids of revolution through an Eulerian modal approach, Journal of Sound and Vibration 331 (4) (2012) 868-882.

[18] O. Montagnier, Tubes composites à grande vitesse de rotation : analyses expérimentales et modélisation, Ph.D. thesis, University of Marseille, France, 2005.

[19] O. Montagnier, E. Chatelet, C. Hochard, G. Jacquet-Richardet, S. Bellizzi, On the experimental identification of damping of carbon/epoxy laminates - Effects on stability of composite rotors, in: Proceedings of the 15th Journées Nationales sur les Composites, Marseille, France, 173-180, 2007.

[20] H. L. Wettergren, On the behavior of material damping due to multi-frequency excitation, Journal of Sound and Vibration 206 (5) (1997) 725-735.

[21] G. Genta, On a Persistent Misunderstanding of the Role of Hysteretic Damping in Rotordynamics, Journal of Vibration and Acoustics 126 (3) (2004) 459-461.

[22] G. Genta, Dynamics of Rotating Systems, Springer, New-York, 2nd edn., 2005.

[23] K. Hibbitt, I. Sorensen, ABAQUS/Standard: User's Manual, Hibbitt, Karlsson \& Sorensen, 2001.

[24] S. Timoshenko, Vibration problems in engineering, D. Van Nostrand company, inc., 1937.

[25] S. Tsai, H. Hahn, Introduction to composite materials, CRC, 1980.

[26] R. D. Adams, D. G. C. Bacon, Effect of fiber orientation and laminate geometry on the dynamic properties of the C.F.R.P., Journal of Composite Materials 7 (1973) 422-428. 
[27] R. G. Ni, R. D. Adams, A rational method for obtaining the dynamic mechanical properties of laminae for prediction of the damping of laminated plates and beams, Composites 15 (3) (1984) 193-199.

[28] O. Montagnier, C. Hochard, Optimisation of hybrid high-modulus/high-strength carbon fibre reinforced plastic composite drive shafts, Materials and design 46 (2013) 88-100, URL http://dx . doi .org/10.1016/j .matdes . 2012 .09.035. 


\section{List of figure captions}

Figure 1: Simply supported axisymmetric tubular composite shaft with rolling-element bearings mounted on viscoelastic supports.

Figure 2: The Campbell diagram of the Zinberg and Symonds' shaft (with $k_{e}=10^{7} \mathrm{~N} \mathrm{~m}^{-1},(-)$ forward modes, $(---)$ backward modes).

Figure 3: Normalized strain distribution in the first mode of a composite with Zinberg and Symonds composite. (a) ABAQUS FEM plate versus laminate theory; (b) ABAQUS FEM cylindrical shell versus laminate theory.

Figure 4: Finite element simulation of the Zinberg and Symonds [14] shaft with ABAQUS: effects of the end fittings on the first natural frequency. (a) First mode of the shaft with thin and infinitely rigid end fittings and detailed mesh of an end fitting; (b) First mode of the shaft with elastic end fittings and detailed mesh of an end fitting.

Figure 5: Normalized strain distribution (a) and stress distribution (b) in the first Zinberg and Symonds' shaft mode: ABAQUS shell versus laminate theory with $\mathbf{B}=\mathbf{0}$.

Figure 6: Evolution of the critical speeds and threshold speed of a composite shaft (T300/5208) mounted on dissipative supports $\left(\left[90,0_{3}, 45,-45\right]_{s}, t_{s}=1.5 \mathrm{~mm}, r_{m}=39.25 \mathrm{~mm}, \eta_{e}=7 \%\right.$ and $\left.m_{b}=1 \mathrm{~kg}\right)$. (a) critical speeds and threshold speed versus the shaft length with $k_{e}=2 \times 10^{6} \mathrm{~N} \mathrm{~m}^{-1}$; (b) critical speeds and threshold speed versus the support stiffness with $l=1.2 \mathrm{~m}$.

\section{List of table captions}

Table 1: Material properties for a 0.6 volume fraction

Table 2: First critical speed in Zinberg and Symonds' case [14] using various theories (Diff. = relative difference in comparison with classical EMBT, Gyr. = gyroscopic effect, R.I. = rotatory inertia, Sh. $=$ transverse shear effect, Sup. $=$ support stiffness effect, Co. $=$ laminate coupling mechanism and E.F. $=$ end fitting)

Table 3: Normalized middle plane strains and curvatures in Zinberg and Symonds composite for the first mode

Table 4: Comparison between ECMBT and EMBT with various stacking sequences in a composite shaft (T300/5208) mounted on dissipative supports $\left(l=2 \mathrm{~m}, t_{s}=1.5 \mathrm{~mm}, r_{m}=39.25 \mathrm{~mm}, k_{e}=2 \times 10^{6} \mathrm{~N} \mathrm{~m}^{-1}, \eta_{e}=7 \%\right.$ and $\left.m_{b}=1 \mathrm{~kg}\right)$ 